\title{
A New Adaptive Fuzzy Hybrid Force/Position Control for Intelligent Robot Deburring
}

\author{
Feng-Yih $\mathrm{Hsu}^{1}$ and $\mathrm{Li}-\mathrm{Chen} \mathrm{Fu}^{1,2}$ \\ Dept. of Electrical Engineering ${ }^{1}$ \\ Dept. of Computer Science \& Information Engineering ${ }^{2}$ \\ National Taiwan University, Taipei, Taiwan, R.O.C.
}

\begin{abstract}
The major control problems for robot deburring mainly arise from uncertainty of the robot manipulators and complex deburring process. In this paper, a new design of hybrid force/position control of robot manipulators via adaptive fuzzy approach is proposed to solve these problems. The control architecture consists of an outer-loop command generator which can automatically determine the desired robot motion profile and an inner-loop adaptive fuzzy hybrid force/position controller which can realtime achieve the command. To demonstrate the effectiveness of the present work, it is applied to the control of a five degree-of-freedom (DOF) articulated robot manipulator for deburring tasks.
\end{abstract}

\section{Introduction}

Applying automated robot manipulators to replace manual deburring operation has become more and more important owing to the high cost of deburring, for some of a variety of cast parts which sometimes amounts to 35 percent of total part's cost [1]. In general, deburring tasks are meant to remove burrs from part's edges and to maintain the final geometry of deburred part's edges within some allowable tolerances. To achieve that, a cutting tool with fixed spindle speed has to chamfer the part edges while undergoing compliant contour-following motion. When driving the cutting tool to perform a deburring task, the deburring robots have to implement two major motions: one is to apply suitable chamfering force to the part's edge so as to remove burrs and to avoid damageto the part, and the other is to perform a contour-following motion to assure that the cutting tool will continue to contact all burrs that spread out over the chamfer.

With an aim to achieving this goal, a heuristic strategy that is often applied by experienced engineers is to control the feedrate of the cutting tool to attain the desired chamfering force. This heuristic is simply slowing down the feedrate of the cutting tool when the encountered burr is large, but speeding it up if otherwise [1], [4].On the other hand, the chamfering force for a constant feedrate and a specific chamfer depth is proportional to the cross sectional area of the burr [2], which motivates us to command the feedrate of the cutting tool so as to control the chamfering force. Human operators, however, can not manipulate the cutting tool so precisely to produce the desired chamfer depth as a deburring robot can. Alternatively, some force control schemes such as impedance control, hybrid force control, and other force control schemes had been applied to compliantly yield a desired chamfer depth [3]-[9]. The common feature of these approaches is to construct a model of the contact force that describes the relationship between the position of the cutting tool and the magnitude of the contact force. However, it is fairly hard to construct a precise model and as a result a deburring scheme based on a humanskill model was proposed [4]. Furthermore, a neural network controller which is equipped with the ability of refining the deburring behavior by learning from the skillful engineers was also developed to solve this problem [5].

To sum up, to achieve automated deburring, the guideline for human operator in performing the deburring task needs to be incorporated into the controller and the self-tuning capability needs to be endowed so as to adaptively correct the control law for coping with uncertainties in deburring dynamics. On the other hand, the fuzzy variable structure control can provide the stability and smoothness at the same time of a fuzzy control if the fuzzy control is formulated in a form of variable structure control [17]-[19], or if a variable structure control is augmented with some ruleparameter setting mechanism [20]-[23]. In this paper, we present an adaptive fuzzy hybrid force/position controller via variable structure control approach to 


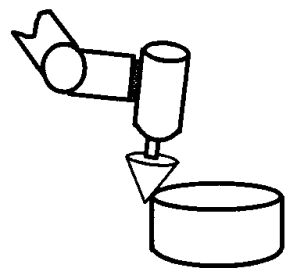

Figure 1: The deburring robot performing the contour-following task

achieve the aforementioned goal in this paper.

\section{Dynamic Model of a Deburring Robot in Cartesian Frame}

Consider an $n$ degree-of-freedom articulated deburring robot whose cutting tool to is commanded to perform contour-following motion in order to remove burrs from a part, as depicted in Fig. 1. Its dynamic model in joint coordinates can be derived as follows:

$$
M(q) \ddot{q}+C(q, \dot{q}) \dot{q}+G(q)+D(\dot{q})=\tau+\tau_{f},
$$

where $q \in \Re^{n}$ is the joint vector, $M(q) \in \Re^{n \times n}$ is the inertia matrix, $C(q, \dot{q}) \dot{q}$ is the vector representing the centrifugal and Coriolis forces satisfying $\dot{M}-2 C$ is a skew-symmetric matrix, $G(q)$ is the vector of gravitational forces, $D(\dot{q})$ is the vector of friction forces, $\tau_{f}$ is the vector of cutting forces and moments, and $\tau$ is the vector of control input forces and moments.

To ease the controller design for the deburring task, we re-express the dynamic model in the Cartesians coordinates. First, we assume that the Cartesians coordinate of the cutting tool, namely, $x$, is with respect to the world frame $\{W\}$, so that $x$ can be represented as a function of its joint coordinates, $q$ in the reference frame, i.e.,

$$
x=H(q) \text {, }
$$

where $x=\left[x_{1}, \cdots, x_{6}\right]^{T}=\left[x_{p}^{T}, x_{o}^{T}\right]^{T}$, with $x_{p} \in \Re^{3}$ being its position vector of cutting tool and $x_{0} \in \Re^{3}$ being the orientation vector, and $q=\left[q_{1}, \cdots, q_{n}\right]^{T}$. Differentiating equation (2), we then get

$$
\dot{x}=\frac{\partial H(q)}{\partial q} \dot{q}=J(q) \dot{q},
$$

where $J(q) \in \Re^{6 \times n}$ is a Jocabin transform matrix and is assumed to be of full rank for $q$ lying in a compact set in the joint space, so that there exists a one-to-one mapping between $x$ and $q$ in a properly defined compact set [11]. Thus, $J$ has a sudo-inverse matrix $J^{+}$, satisfying $J J^{+}=I$. Then, letting $M_{x}=J^{+T} M J^{+}$, $C_{x}=J^{+T} C J^{+}-J^{+T} M J^{+} \dot{J} J^{+}, G_{x}=J^{+T} G, D_{x}=$ $J^{+T} D, f_{\tau}=J^{+T} \tau$ and $f=J^{+T} \tau_{f}$, we can derive the dynamics of the robot manipulator in the world frame as follows:

$$
M_{x}(x) \ddot{x}+C_{x}(x, \dot{x}) \dot{x}+G_{x}(x)+D_{x}(\dot{x})=f_{\tau}+f,
$$

Here, the torque vector $\tau$ in joint coordinates can be derived as $\tau=J^{T} f_{\tau}$. To simplify the underlying control problem, we will assume that the cutting tool is in contact with the part only at a single point so that the moment exerted by the cutting tool equals zero. Therefore, the cutting force/moment, $f$, can be denoted as $f=\left[f_{n}^{T}+f_{t}^{T}, 0,0,0\right]^{T}$, where $f_{n} \in \Re^{3}$ is the vector of contact force perpendicular to the chamfer surface of the part, and $f_{t} \in \Re^{3}$ is the vector of the chamfering force tangential to the chamfer contour of the part.

Now, we need to derive the dynamics of the chamfering force and the contact force during the deburring process. Let $x_{s}$ represent the vector of the cutting tool position on the part's edge when the chamfer depth is zero (see Fig. 2), namely, $x_{s}$ is subject to a constraint equation $\phi\left(x_{s}\right)=0$. To express $f_{n}$ and $f_{t}$ on the chamfer surface, we define two unit orthogonal vectors, $n_{1}$ and $n_{2}$. The former is opposite to the direction of the contact force and can be constructed as $n_{1}=\frac{-\nabla \phi\left(x_{s}\right)}{\left\|\nabla \phi\left(x_{s}\right)\right\|}$, where $\nabla$ represents a gradient operator. However $n_{1}$ can not be constructed directly from the above equation since $x_{s}$ is not measurable. Therefore, we approximate $n_{1}$ by $n_{1} \cong \frac{-\nabla \phi\left(x_{p}\right)}{\left\|\nabla \phi\left(x_{p}\right)\right\|}$, when the chamfer depth, say, $\delta \in \Re^{1}$, very small, where $x_{p}$ is defined to be the position of the cutting tool, which is attributed to the fact that $\nabla \phi\left(x_{p}\right) \times \nabla \phi\left(x_{s}\right) \cong 0$. On the other hand, $n_{2}$ is defined to be opposite to the direction of contour-following motion on a chamfer with zero depth, satisfying $n_{1} \perp n_{2}$, then it can be expressed as

$$
n_{2}=\frac{\dot{x}_{p}-n_{1} n_{1}^{T} \dot{x}_{p}}{\left\|\dot{x}_{p}-n_{1} n_{1}^{T} \dot{x}_{p}\right\|}=\frac{\left(I-n_{1} n_{1}^{T}\right) \dot{x}_{p}}{\left\|\left(I-n_{1} n_{1}^{T}\right) \dot{x}_{p}\right\|}
$$

As a result, we can rewrite the contact force and the chamfering force, respectively, as $f_{n}=-\left\|f_{n}\right\| n_{1}$ and $f_{t}=-\left\|f_{t}\right\| n_{2}$. Furthermore, it is shown that the magnitude of $f_{t}$ is found to satisfy the following [4]:

$$
\begin{aligned}
\left\|f_{t}\right\| & =g\left|n_{2}^{T} \dot{x}_{p}\right|+h\left|n_{1}^{T} \dot{x}_{p}\right|+P_{t h}^{\prime} \\
& =g\left\|\left(I-n_{1} n_{1}^{T}\right) \dot{x}_{p}\right\|+h\left|n_{1}^{T} \dot{x}_{p}\right|+P_{t h}^{\prime},
\end{aligned}
$$

where $g$ and $h$ are coefficients which vary with different burrs and chamfer depth, and $P_{t h}^{\prime}$ is a constant value representing the threshold power of the cutting tool. However, it is very much difficult to establish an exact model for the contact force. Here, we assume that the deburring robot is rigid enough and the spindle of the cutting tool rotates at fast speed, so that the magnitude of the contact force will roughly obey the following relations:

$$
\left\|f_{n}\right\|=\left\{\begin{array}{cl}
c_{2} \dot{\delta}+c_{1} \delta+c_{0}, & \text { as } \delta>=0 \\
0, & \text { otherwise }
\end{array}\right.
$$



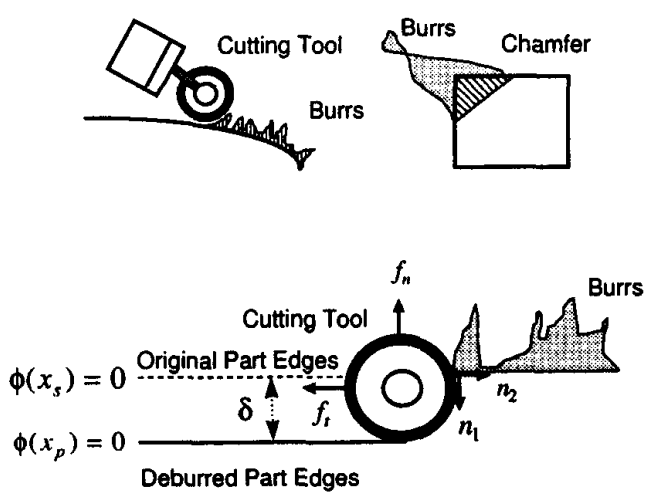

Figure 2: The geometry of part edges durring the deburring process

where $c_{0}$ is a threshold as the cutting tool contacts the chamfer, $c_{1}$ is the stiffness of the part, and $c_{2}$ is the damping ratio of the cutting tool moving into the chamfer.

\section{Control Architecture of the Deburring Robot}

Thus far, we have derived the dynamics of the robot which performs deburring operation. To accomplish the deburring task, a well designed controller to drive the robot system is needed. Here, the proposed control architecture consists of an outer-loop command generator and an inner-loop controller, as depicted in Fig. 3. The former determines a positional profile command such that the desired chamfering force and contour-following motion can be realizable, whereas the latter aims at driving the robot to execute that command in a compliant manner so as to yield a desired chamfer depth. Generally speaking, since the servo-rate of the inner-loop controller is much faster than that of the outer-loop command generator, we assume that the outer-loop has a nonzero servo-period $T$ whereas the inner-loop controller is considered as a continuous type for simplicity, i.e. its servo-period is zero. The motion commands which are not so much time critical from the viewpoint of the inner-loop controller, like the chamfering force and the locus of the contour-following motion, are handled in the outerloop. The positional command from the command generator is then transferred to a continuous input via passing through a first-order hold mechanism for the inner-loop controller.

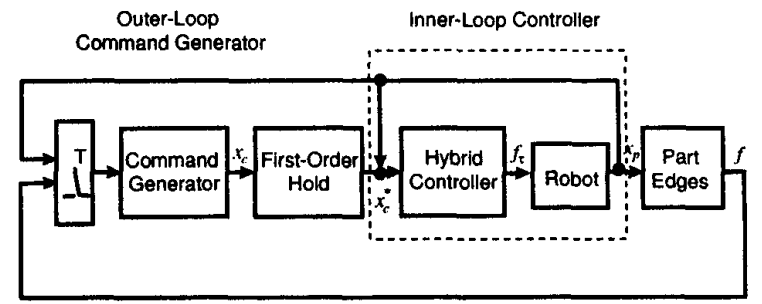

Figure 3: The control architeture of the deburring robot

\subsection{Outer-Loop Command Generator}

The function of the outer-loop command generator is mainly to ask the inner-loop controller to control the attached cutting tool to produce a desired positional profile. Hence, our goal here is to design such a desired position command, whereby the following objectives can be simultaneously realized provided the cutting tool can implement the command.

- maintaining a desired constant chamfering force

- performing a contour-following motion

First, simplifying the model of the chamfering force as in equation (6), we consider the case where the cutting tool velocity in the direction of $n_{1}$ is much smaller than that in the direction of $n_{2}$, i.e., $\left|n_{1}^{T} \dot{x}_{p}\right| \ll<$ $\left|n_{2}^{T} \dot{x}_{p}\right|$. As much, we let $\left\|f_{t}\right\|$ tend to a constant $\left\|f_{t_{d}}\right\|$ by letting the cutting tool position $x_{p}$ follow a desired positional profile command $x_{c}$ during the sampled-period, expressed as follows:

$$
x_{c}(k T)=\left\{\begin{array}{l}
x_{p}(k T)+\frac{\left\|n_{2}^{T} \dot{x}_{p}(k T)\right\|\left(\left\|f_{t}\right\|-P_{t h}^{\prime}\right)}{\left\|f_{t}(k T)\right\|-P_{t h}^{\prime}} T n_{2}(k T) \\
\text { if }\left\|f_{t}(k T)\right\|-P_{t h}^{\prime}>v_{\epsilon} ; \\
x_{p}(k T)+v_{0} T n_{2}(k T), \quad \text { otherwise, }
\end{array}\right.
$$

where $T$ is the servo-period of the outer loop, $k=$ $1,2, \cdots ; v_{0}$ is the default feedrate, set to prevent $x_{p}$ from changing too fast to damage the part as $\left\|f_{t}\right\|-P_{t h}^{\prime}$ becomes excessively small, where $v_{\epsilon}>0$ is its offset. Clearly, when $\dot{x}_{p} \rightarrow \frac{x_{c}(k T)-x_{c}(k T-T)}{T}$, we can get a result that $\left\|f_{t}\right\|$ closely approximates $\left\|f_{t_{d}}\right\|$.

Finally, passing $x_{c}(k T)$ through a modified firstorder hold filter to the inner-loop controller, we can get a continuous desired position trajectory $x_{c}^{*}(t)$ with an initial value $x_{c}^{*}(0)=x_{c}(0)$ as follows:

$$
x_{c}^{*}(t)=x_{c}{ }^{*}(k T)+\frac{x_{c}(k T)-x_{c}^{*}(k T-T)}{T}(t-K T)
$$




\subsection{Inner-Loop Hybrid Force/Position Controller}

The inner-loop controller is to drive the robot to perform the aforementioned positional profile command in a compliant manner so as to yield a desired chamfer depth. Since the direction for measure of the desired chamfer depth is perpendicular to that of the contour-following motion, we can apply a hybrid force/position control to achieve the above goal.

In order to obtain the desired chamfer depth, denoted as $\delta_{d}^{*}$, under the desired contact force, denoted as $f_{n d}$, the desired positional trajectory of the cutting tool $\delta_{d}(t)$, in the direction of the chamfer depth, i.e., $n_{1}$ needs to be carefully selected. Apparently, $\delta_{d}(t)$ is related to the contact force $f_{n}$, and it will eventually approach the desired chamfer depth $\delta_{d}^{*}$ provided the contact force approaches the desired one. To construct $\delta_{d}(t)$, first we let the magnitude of desired contact force $f_{n_{d}}$ be defined as follows:

$$
\left\|f_{n_{d}}\right\|=\left(c_{1} \delta_{d}^{*}+c_{0}\right)
$$

Then, $\delta_{d}(t)$ is defined such that its initial condition $\delta_{d}(0)=0$ and its time derivative is given in the following:

$$
\begin{aligned}
\dot{\delta}_{d} & =k_{\delta}\left(\left\|f_{n_{d}}\right\|-\left\|f_{n}\right\|\right)=k_{\delta}\left[-c_{2} \dot{\delta}+c_{1}\left(\delta_{d}^{*}-\delta\right)\right] \\
& =k_{\delta} e_{f},
\end{aligned}
$$

where $k_{\delta}>0$ and $e_{f}=\left\|f_{n_{d}}\right\|-\left\|f_{n}\right\|$ is defined as the current error of the contact force. Note that, when $\dot{\delta} \rightarrow \dot{\delta}_{d}$ and $\delta \rightarrow \delta_{d}$, we can rewrite the above equation as

$$
\left(1+k_{\delta} c_{2}\right) \dot{\delta}_{d}+k_{\delta} c_{1} \delta_{d}=k_{\delta} c_{1} \delta_{d}^{*}
$$

which implies that $\delta_{d}$ approaches $\delta_{d}^{*}$ exponentially and, hence, the error of the contact force, $e_{f}$, can approach zero.

Given $x_{c}^{*}(t)$ and $\delta_{d}$, we can augment the contourfollowing position command by summing the desired chamfer depth trajectory $\delta_{d} n_{1}$ and the desired contour-following profile trajectory $x_{c}^{*}$ together. Thus, an augmented desired position trajectory, $x_{p_{d}}$, can be expressed as

$$
x_{p_{d}}(t)=x_{c}^{*}(t)+\delta_{d}(t) n_{1},
$$

from which we now define the position tracking error as $e_{p}(t)=x_{p_{d}}-x_{p}$. Similarly, we let the desired orientation of the cutting tool be given as $x_{o d}$ so that the collective position/orientation trajectory can be denoted as $x_{d}=\left[x_{p_{d}}^{T}, x_{o d}^{T}\right]^{T}$. By this definition, we now define the vector of tracking position/orientation tracking error vector as $e(t)=x_{d}(t)-x(t)=\left[e_{p}^{T}, e_{o}^{T}\right]^{T}$, where $e_{o}=x_{o d}-x_{o}$, which facilitates us to rewrite the dynamic model of the manipulators system (4) as follows:

$$
M_{x}(x) \ddot{e}=M_{x} \ddot{x}_{d}+C_{x}(x, \dot{x}) \dot{x}+G_{x}(x)+D_{x}(\dot{x})-f_{\tau}-f,
$$

Clearly, our objective is now transferred to the design of a control law $f_{\tau}$ that can drive $e$ to zero so that the desired (constant) chamfering force $f_{t_{d}}$ and the desired (constant) chamfer depth $\delta_{d}^{*}$ can be achieved simultaneously. To that end, we define a sliding variable vector $s=\dot{e}+\lambda e$, where $\lambda \in \Re^{6 \times 6}$ is a diagonal positive definite matrix, and re-express the above dynamic model (13) as follows:

$$
\begin{aligned}
M_{x} \dot{s}+\left(K+C_{x}\right) s= & M_{x}\left(\ddot{x}_{d}+\lambda \dot{e}\right)+G_{x}+D_{x}+C_{x} \\
& \left(\dot{x}_{d}+\lambda e\right)+K s-f-f_{\tau},
\end{aligned}
$$

for some diagonal positive matrix $K \in \Re^{6 \times 6}$. Apparently, if the controller design can force the right hand side (RHS) of the equation (14) to be zero, then the resulting system will be subject to the closed-loop dynamics $M_{x} \dot{s}+\left(K+C_{x}\right) s=0$, which not only assures the stability of the system, but also guarantees the exponential convergence of the tracking error $e$. As a result, an ideal control law can be designed as

$$
f_{\tau}^{*}=M_{x}\left(\ddot{x}_{d}+\lambda \dot{e}\right)+G_{x}+D_{x}+C_{x}\left(\dot{x}_{d}+\lambda e\right)+K s-f
$$

However, it is difficult to implement this ideal controller due to various kinds of model uncertainty and, hence, the major control problem here is how to realize such an ideal controller.

\section{Control Algorithm}

As has been mentioned, the parameters associated with contact force, $c_{0}, c_{1}$ and $c_{2}$ are often estimated as $\widehat{c}_{0}, \widehat{c}_{1}$, and $\widehat{c}_{2}$, respectively. These estimate values will result in the estimate of the desired contact force apart from the true desired one, and will be referred to as the virtual desired force in contrast with the actual desired one. Denote this virtual desired contact force, $\widehat{f}_{n_{d}}$, whose magnitude can be expressed as

$$
\left\|\widehat{f}_{n_{d}}\right\|=\widehat{c}_{0}+\widehat{c}_{1} \delta_{d}^{*}
$$

Apparently, here our goal is to let $\left\|\widehat{f}_{n_{d}}\right\|$ approach $\left\|f_{n_{d}}\right\|$ so that $\delta_{d}(\infty)=\delta_{d}^{*}$. To achieve this goal, we build a virtual contact force $\widehat{f}_{n}$ and express its magnitude as

$$
\left\|\widehat{f}_{n}\right\|=\widehat{c}_{0}+\widehat{c}_{1} \delta+\widehat{c}_{2} \dot{\delta}
$$

whereby the error between $\left\|f_{n}\right\|$ and $\left\|\widehat{f_{n}}\right\|$ can be derived as follows:

$$
e_{n}=\left\|f_{n}\right\|-\left\|\hat{f}_{n}\right\|=\tilde{c}_{0}+\widetilde{c}_{1} \delta+\tilde{c}_{2} \dot{\delta},
$$

where $\widetilde{c}_{0}=c_{0}-\widehat{c}_{0}, \widetilde{c}_{1}=c_{1}-\widehat{c}_{1}$ and $\widetilde{c}_{2}=c_{2}-\widehat{c}_{2}$. To reduce this error, we update the parameters by the following update law:

$$
\dot{\hat{c_{0}}}=r e_{n} ; \dot{\hat{c_{1}}}=r \delta e_{n} ; \quad \dot{\hat{c_{2}}}=r \dot{\delta} e_{n},
$$

for some $r>0$.

Consider the robust controller as following:

$$
\begin{aligned}
f_{\tau} & =K s-f+H(\|x\|, s) \\
H & =\left(b_{1}+b_{2}\|x\|+b_{3}\|x\|^{2}\right) \operatorname{sgn}(s)+\left(b_{4}+b_{5}\|x\|\right) s,(20)
\end{aligned}
$$

where $b_{1}, \cdots$, and $b_{5}$ are some constants. Then, the following theorem is valid.

Theorem 4.1 If the control law is given as in (20), then the tracking error $e$ will be driven to zero exponentially in term $t$. 


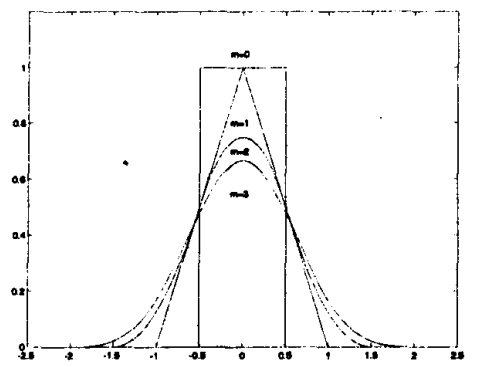

Figure 4: The m-order B-spline basis for $m=0,1,2$, and 3

\subsection{Adaptive Fuzzy Hybrid Control}

Howecer, gain parameters $b_{1}, \ldots, b_{5}$ of $H(\|x\|, s)$. are very difficult to be obtained. Here, we introduce an adaptive fuzzy control algorithm as our control solution. Let the actual control law $f_{\tau}$ be rewritten as follows:

$$
f_{\tau}=K s-f+f_{s}(\|x\|, s),
$$

where $K s$ is a linear PD type compensator and $f_{8}$ is the key adaptive fuzzy compensator. Here, our goal is to design a fuzzy controller $u_{f}=\left[u_{f_{1}}, \cdots, u_{f_{n}}\right]^{T}=f_{s}$ which can compensate for the uncertainties. Then, consider a fuzzy controller $u_{f}$, consisting of $n$ ( $n=$ 6) two-input single-output (MISO) fuzzy controllers, which are respectively characterized by

$$
u_{f_{i}} \triangleq u_{f_{i}}\left(\|x\|, s_{i}\right) \equiv u_{f_{i}}\left(y_{0}, y_{i}\right): \Omega_{0} \times \Omega_{i} \rightarrow \Re
$$

where $u_{f_{i}}$ is the $i$-th fuzzy controller, $y=$ $\left[y_{0}, y_{1}, \cdots, y_{n}\right]^{T}=\left[\|x\|, s_{1}, \cdots, s_{n}\right]^{T}$ and $\|x\|$ and $s_{i}$ (i.e., $y_{0}$ and $y_{i}$ ) are the $i$-th input fuzzy variables, $\Omega_{0} \equiv\left[-\Upsilon \Delta_{0}, \Upsilon \Delta_{0}\right], \cdots, \Omega_{n} \equiv\left[-\Upsilon \Delta_{n}, \Upsilon \Delta_{n}\right]$ with $\Upsilon$ being a positive integer and can be set arbitrarily large to constitute enlarge enough compact sets, and $\Delta_{0}, \cdots, \Delta_{n}$ being some positive real numbers. Here, each of the membership functions is given as an $m$ th order multiple dimension central B-spline function (as depicted in Fig.4), of which the $k$-th dimension is defined as follows:

$N_{m k}\left(y_{k}\right)=\sum_{l=0}^{m+1} \frac{(-1)^{l}}{m !}\left(\begin{array}{c}m+1 \\ l\end{array}\right)\left[\left(y_{k}+\left(\frac{m+1}{2}-l\right) \Delta_{k}\right)_{+}\right]^{m}$

where we use the notation

$$
x_{+}:=\max (0, x)
$$

The $m$-th order B-spline type of membership function has the following properties:

- an $(m-1)$-th order continuously differentiable function, i.e., $N_{m k}\left(y_{k}\right) \in C^{m-1}$;

- local compact support, i.e., $N_{m k}\left(y_{k}\right) \neq 0$ only for $y_{k} \in\left[-\frac{m+1}{2} \Delta_{k}, \frac{m+1}{2} \Delta_{k}\right]$

- $N_{m k}\left(y_{k}\right)>0$ for $y_{k} \in\left(-\frac{m+1}{2} \Delta_{k}, \frac{m+1}{2} \Delta_{k}\right)$
- symmetric with respect to the center point (zero point)

- $\sum_{i=-\infty}^{\infty} \sum_{j=-\infty}^{\infty} N_{m 0}\left(y_{0}-i \Delta_{0}\right) N_{m k}\left(y_{k}-j \Delta_{k}\right)=$ 1 , for $k \in \mathcal{Z}^{+}$

Then the membership functions for the $k$-th fuzzy variable $y_{k}$ are defined as follows:

$$
\mu_{k_{i}}\left(y_{k}\right)=N_{m k}\left(y_{k}-i \Delta_{k}\right)
$$

whose compact support is given as:

$$
\Omega_{k i}=\left[\left(i-\frac{m+1}{2}\right) \Delta_{k},\left(i+\frac{m+1}{2}\right) \Delta_{k}\right],
$$

for $k=0, \cdots, n$, and $i=-\Upsilon, \cdots, 0, \cdots, \Upsilon$, which means that $y_{k} \in \operatorname{int}\left(\Omega_{k_{i}}\right)$ implies that $\mu_{k_{i}}\left(y_{k}\right)>0$ and $\Omega_{k} \equiv \cup_{i \in\{-\Upsilon, \cdots, \Upsilon\}} \Omega_{k i}$. Apparently, it is possible that $\Omega_{k_{i}} \cap \Omega_{k_{j}} \neq \emptyset$, for some $i \neq j$, i.e., $y_{k}$ can simultaneously fall into several compact supports. From [15], we can represent the above fuzzy controllers as follows:

$$
\begin{aligned}
u_{f_{k}} & =\frac{\sum_{i=-\Upsilon}^{\Upsilon} \sum_{j=-\Upsilon}^{\Upsilon} \mu_{0_{i}}\left(y_{0}\right) \mu_{k_{j}}\left(y_{k}\right) \theta_{k(i j)}}{\sum_{i=-\Upsilon}^{\Upsilon} \sum_{j=-\Upsilon}^{\Upsilon} \mu_{0 i}\left(y_{0}\right) \mu_{k j}\left(y_{k}\right)} \\
& =\sum_{i=-\Upsilon}^{\Upsilon} \sum_{j=-\Upsilon}^{\Upsilon} \nu_{k(i j)}\left(y_{0}, y_{k}\right) \theta_{k(i j)}=\theta_{k}{ }^{T} \nu_{k},
\end{aligned}
$$

where $i, j$ are integer indices, $\nu_{k(i j)}\left(y_{0}, y_{k}\right)$ is the fuzzy basis function, $\theta_{k(i j)}$ is the parameter, $\theta_{k}=$ $\left[\theta_{k(-\Upsilon-\Upsilon)}, \cdots, \theta_{k(00)}, \cdots, \theta_{k(\Upsilon \Upsilon)}\right]^{T} \in \Re^{(2 \Upsilon+1)^{2}}$ and $\nu_{k}=\left[\nu_{k(-\Upsilon-\Upsilon)}, \cdots, \nu_{k(00)}, \cdots, \nu_{k(\Upsilon \Upsilon)}\right]^{T} \in \Re^{(2 \Upsilon+1)^{2}}$.

Define the $k$-th element of a new vector $y_{\Delta}=$ $\left[y_{\Delta_{0}}, \cdots, y_{\Delta_{n}}\right]^{T}$ as follows:

$$
y_{\Delta_{k}}= \begin{cases}y_{k}, & \text { as } y_{k}<-\Delta_{k} \text { or } y_{k}>\Delta_{k} ; \\ 0, & \text { otherwise (i.e. } \left.y_{k} \in\left[-\Delta_{k}, \Delta_{k}\right]\right)\end{cases}
$$

so that

$$
\dot{y}_{\Delta_{k}}=\dot{y}_{k} \text { for } y_{\Delta_{k}} \neq 0 \text {. }
$$

Here, our goal is to design $u_{f}$ to satisfy the following

$$
\begin{gathered}
u_{f_{k}}\left(\|x\|, s_{k}\right)= \begin{cases}k_{f_{k}}(\|x\|) \operatorname{sgn}\left(s_{k}\right), & \text { if } s_{k} \notin\left[-\Delta_{k}, \Delta_{k}\right] ; \\
k_{s_{k}}\left(\|x\|, s_{k}\right), & \text { otherwise; }\end{cases} \\
\equiv u_{f_{k}}\left(y_{0}, y_{k}\right)= \begin{cases}k_{f_{k}}\left(y_{0}\right) \operatorname{sgn}\left(y_{k}\right), & \text { if } y_{k} \notin\left[-\Delta_{k}, \Delta_{k}\right] ;(28) \\
k_{s k}\left(y_{0}, y_{k}\right), & \text { otherwise; }\end{cases}
\end{gathered}
$$

where $k_{f}(\|x\|, s) \geq|H(\|x\|, s)|, \quad k_{s}(\|x\|, s)=$ $\left[k_{s 1}, \cdots, k_{s n}\right]^{T}$ is a smooth function vector to make $u_{f}$ smooth, and $\left[-\Delta_{k}, \Delta_{k}\right]$ is regarded as a designated dead-zone range which can be arbitrarily set.

Then the following adaptive law to update the parameters vector $\theta_{k}$ will be necessary so that the tracking error can be driven toward the dead-zone range:

$$
\dot{\theta}_{k(i j)}=r \nu_{k(i j)}\left(y_{0}, y_{k}\right) y_{\Delta_{k}}, \text { for } y_{k} \in \Omega_{k}
$$

where $r>0$ is some positive constant.

Finally, we design $f_{\tau}$ as

$$
f_{\tau}=K s-f+\operatorname{diag}(\zeta) f_{s}(\|x\|, s)+[I-\operatorname{diag}(\zeta)] \bar{H}(\|x\|, s) \quad(30)
$$




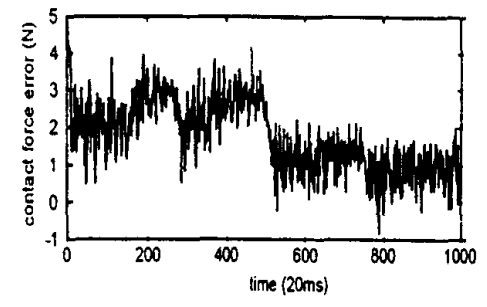

Figure 5: The tracking error of normal contact force

where $\operatorname{diag}(\bar{H}) \operatorname{sgn}(s)>\operatorname{diag}(H) \operatorname{sgn}(s)$, and the $k$-th element of $\zeta \in \Re^{6}$ is defined as follows:

$$
\zeta_{k}= \begin{cases}1, & \text { as } y_{k} \in \Omega_{k} \\ 0, & \text { otherwise. }\end{cases}
$$

Then, the following theorem states the condition under which the above-mentioned adaptive fuzzy control law will yield satisfactory result.

Theorem 4.2 If the update law and the control law are given as in equations (29) and (30), then the tracking errors will asymptotically converge to a neighborhood of zero.

\section{Experimental Results}

A five degree-of-freedom (DOF) articulated deburring robot arm equipped with a Zebra force sensor and a cutting tool is set up in the Intelligent Robot Laboratory of Dept. of Computer Science \& Information Engineering in National Taiwan University. The experiment is performed by controlling the robot arm to drive its cutting tool to chamfer the edge of the cylinder part which has a 30mm.radius for cross section. The control architecture based on an industrial PC ( $80486 \mathrm{dx} 2-66 \mathrm{CPU}$ ) has $2.5 \mathrm{~ms}$ sampling-period for the inner loop hybrid controller and $25 \mathrm{~ms}$ sampling period for the outer loop command generator. The desired contact force and the desired chamfering force are set to $5 \mathrm{Nt}$ and $10 \mathrm{Nt}$, respectively. The number of fuzzy rules is set to equal $6 \times(2 \Upsilon+1)^{2}=6 \times 9 \times 9$. At the beginning, since initial parameters in matrix $\theta$ are set to zeros, which is similar to only use the PD controller to compensate for the uncertainties in the first period of deburring motion after the first period the force error is quickly driven toward zero as Fig. 5.

\section{Conclusions}

We had proposed an adaptive fuzzy hybrid force/position controller, which can update fuzzy rules to compensate for the robot dynamics along with the force dynamics induced by the contact between the cutting tool and the part's edge and identify the actual desired contact force. Various experimental results have been provided to verify the effectiveness of the developed work.

References

[1] H. Kazernooi, J. J. Kramer and B. M., "An approach to Automated Deburring by Robot Manipulators," ASME Journal of Dynamic Syetems Mensusements
And Control, pol. 108. no.4. DEC. 1986

[2] M. G. Her and H. Kaxernooi, "Automated Robotic Deburring of Parts Using Compliance Control," ASME Journal of Dynamie Systeme Measurements
And Control. vol. 113. March. 1991

[3] H. Kaxernooi, "On the Robot Compliant Motion Control," ASME Journal of Dynamic Systems Meisurements And Control. whl. 111. September, 1989

[4] Sheng Lu and H. Aanda, "Transferring Manipulative Skills to Robots: Representation and Acquisition of Tool Manipulative Skills Using a Proceso
Dynamics Control," ASME Joutunl of Dynamic Syetems Mensurements And ConDynamics Control," AS
trmi. tol. 114. June. 1992

(5) Sheng Lu and H. Asada, "Teaching and Learning of Deburring Robots Using Neural Networks," Proc. Of 1993 IEEE Int. Conf. On Robotics and AtUsing Neural Netwo

[6] G. M. Bone and M. A. Elbestawi " Robotic Force for Deburring Using an Active End Effector," Roboticn. val. 7. pp-303-308. 1989

[7] G. M. Bone, M. A. Elbestawi, R. Lingarkar and 1. Liu "Force Control for Robotic Deburring:" ASME Journal of Dynamic Systeman Mensurements And Cantral, mal. 113. Septemiber, pp.395-400.1991

[8] M. S. Ali, M. N. Noori and J. Turi, "Automatic Deburring Utilizing Real-Time Impedance Control Strategy," Computer and Strueture vol. 46. no. Real-Time Impsed

191 D. Jeon and M. Tomizuka. "Lenrning Hybrid Force and Position Control of Robot Manipulators," IEEE Transuctions on Robotics and Automation un.

[10] L. X. Whng. "Stable Adnptive Fuzzy Control of Nonlinenr Systems", IEEE. Conf. on Decision and Control. 1992

[11] J. Maraden, Elementary Classical Analysis. San Franciso: W. H. Freeman 1974

[12] J-J E. Slotine and W. Li, Applied Nonlitent Control, Englewood Cliffs, NJ: Prentice Hall, pp. 278-284, 1991.

[13] R. M. Sanner and J-J E. Slotine, "Gaussian Networks for Direcet Adaptive Control". IEEE. Twnis. on Nenrnl Netrorks, vol. 3. no. 6. pp. 837-868. 1092

[14] S. Sastry and M. Bodson, Adaptive Control: Stability. Comwergence, and Robutetness, Prentice Hall, 1989.

(15] L.-X. Wang, Adnptive Furzy Systems and Control: Design and Stability analysis, NJ:Prentice Hall, 1994

[16] V. S. C. Raviraj and P. C. Sen. "Comparative Study of ProportionalIntegral, Sliding Mode. and Fuszy Logic Controllers for Power Converters,"
IEEE Trans. on Indtutry Applications, vol. 39. no. 2, pp. 518.524. 1997

[17] Jacob S. Glower and Jeffery Muunighan, "Designing Fuzzy Controllers from a variable structures

[18] S.-C. Lin and Y.-Y. Chen, "Design of Self-Learning Fuzxy Sliding Mode Controllers Baned on Genetic Algorith ms." Fuzzy Sets and Systems vol.86. no.2. pp. 1.99-15.9. Mar. 1997

[19] J. C. Wu and T. S. Liu, "Fuzzy Control Stabilization with Application to Motorcycle Control," IEEE Tmans. on Systems. Man. and Cybernstics. PartiB Cybernetics. "ol, 26, no. 6. pp.8.46-847. Der. 1996

[20] Feng-Yih Hsu and Li-Chen Fu, "Adaptive Robust Fuxzy Control for Robo (E⿱

[21] Feng-Yih Hou and Li-Chen Fu, "A New Design of Adaptive Furzy Hybrid Force/Position Controller for Robot Manipulators", IEEE Conferenne. on Robotick and Automution. pp. 869.868, 1995

[22] Feng-Yih Hsu and Li-Chen Fu, "An Adaptive Fuzzy Hybrid Control for Robot Manipulators Following Contours of an Uncertain Object", IEE

[23] Feng-Yih Hsu and Li-Chen Fu, "Intelligent Robot Deburring Using Adaptive Fuzzy Hybrid Control" Proc. 27th International Sympositum on Industrial tive Fuzzy Hybrid Control" Proc.
Robots. pp. 84 Y.852, Milan. Italy. 1996

[24] Feng-Yil. Hsu and Li-Chen Fu, "Recent Progress in Fuzzy Control," A Chapter in Control Problems in Robotics and Automation: Future Diree
tions. Ed. Bruno Sicilliano, Springer-Verlag. London, 1997 